\title{
Editorial: Ludii, Konane and the Computer Olympiads
}

\author{
Tristan Cazenave \\ LAMSADE, Université Paris-Dauphine, PSL, CNRS, Paris, France \\ E-mail:Tristan.Cazenave@dauphine.psl.eu
}

The first scientific contribution to this issue is Deep Learning for General Game Playing with Ludii and Polygames by Dennis J.N.J. Soemers, Vegard Mella, Cameron Browne and Olivier Teytaud. Polygames is a general Deep Reinforcement Learning engine that combines Monte Carlo Tree Search and Deep Neural Networks trained through self-play. Ludii is a general game system that now contains more than one thousand games. The paper describes the bridge between Ludii and Polygames that enables Polygames to train and evaluate games that are implemented and run through Ludii. Polygames can represent any game implemented in Ludii. The authors also give experimental results for multiple board games and discuss future research.

The second scientific contribution is Solving Narrow Konane Boards by Jos W.H.M. Uiterwijk. Konane is a partizan combinatorial game involving captures. The independent subgames can be associated to numbers and nimbers that can be summed to get the value of the whole game combining the subgames. The paper explains two notions that proved very useful to solve Konane boards. The safe moves of a player are the moves that cannot be prevented by the opponent. The independence of two fragments relies on the impossibility of their interaction. Using these notions some results on solving $1 \times n, 2 \times n, 3 \times n$ and $4 \times n$ boards are found.

In this issue we also have a report by Jonathan Schaeffer on the Computer Olympiads from 1989 up to now.

Tristan Cazenave 